\title{
ON THE REMOVAL OF FOUR TERMS FROM AN EQUATION BY MEANS OF A TSCHIRNHAUS TRANSFORMATION*
}

\author{
BY RAYMOND GARVER
}

The removal of the second, third, and fourth terms from an equation $f(x)=0$ by means of a Tschirnhaus transformation is a matter which can be summarized in two statements. First, if $f(x)=0$ is of higher than the fourth degree, the well known Jerrard process, which uses a fourth degree transformation, serves to remove the three terms without requiring the solution of any equation of degree greater than three. Second, if $f(x)=0$ is of the fourth degree, Lagrange first showed that a similar reduction is possible, employing a third-degree transformation. $\dagger$ Lagrange's transformation does not, however, apply to higher degree equations; the fact that the transformed quartic is a binomial equation is essential to its success.

The removal of the second, third, fourth, and fifth terms leads to a larger number of different cases. In general, we can say that, since the equations of condition are of degree $1,2,3,4$, we may be led to an equation of the 24 th degree. But this maximum can almost always be substantially reduced. Thus Hamilton, in his report on the validity of Jerrard's work, was able to show that, if $f(x)=0$ is of degree greater than ten, the transformation can be effected with the aid of a single quartic equation, and no equation of higher degree, while simply one additional quartic has to be introduced if $f(x)=0$ is of degree ten. $\ddagger$ Sylvester later obtained

* Presented to the Society, December 27, 1928.

† See his Oeuvres, vol. III, Paris, 1869, pp. 284-295. The article, his well known Réflexions sur la résolution algébrique des equations, was first published in 1770-1771.

¥ Sixth Report of the British Association for the Advancement of Science, London, 1837, pp. 295-348. See, in particular, p. $318 \mathrm{ff}$. 
these same results in a somewhat different manner.* Bohlin professed to show that the transformation for $f(x)=0$ of degree ten required only one quartic equation, $\dagger$ but Netto $\ddagger$ and Heymann $\S$ pointed out that his work was incorrect. Heymann also set up a very simple transformation for the removal of the first four intermediate terms which requires a single quartic, and no equation of higher degree, but it can be applied only if $f(x)=0$ is of at least the twelf th degree. The only other work on the subject seems to be that of Lagrange in connection with the quintic; $\|$ he showed that the four intermediate terms can be removed without requiring the solution of any equation of higher than the sixth degree. But his method applies only to the quintic, since it makes use of the fact that the transformed equation is binomial.

The purpose of the present paper is to consider the cases which do not seem to be covered in the literature, namely, those in which the equation $f(x)=0$ is of degree $6,7,8$ or 9 . The following theorem gives the principal result:

THEOREM. The second, third, fourth, and fifth terms of a ninth degree equation can be removed by means of a Tschirnhaus transformation of the eighth degree, the determination of whose coefficients involves the solution of no equation of degree greater than five.

The method used to obtain this result was suggested by an article of Hilbert. 9 However, the theorem does not at all follow from his work, which leads to an equation of the twentyseventh degree. In fact the aim of Hilbert's paper is simply to show that the general ninth degree equation is solvable in terms of algebraic functions of not more than four arguments, in the same sense that the general quintic is solvable

* Journal für Mathematik, vol. 100 (1887), pp. 465-486.

$\dagger$ Arkiv för Matematik, Astronomi, och Fysik, vol. 3 (1906-7), No. 5.

$\ddagger$ Grunert's Archiv, (3), vol. 14 (1909), pp. 125-126.

\& Grunert's Archiv, (3) vol. 19 (1912), pp. 320-330.

|| Pp. 314-317 of the above reference.

If Mathematische Annalen, vol. 97 (1926), pp. 243-250. 
in terms of functions of a single argument. The point of view is thus quite different from that of the present paper.

To remove the second, third, fourth, and fifth terms from an equation $f(x)=0$ of the ninth degree by a Tschirnhaus transformation $y=P(x)$, where $P(x)$ is a polynomial of not higher than the eighth degree, we must show that the coefficients of $P(x)$ can be chosen so as to make $\sum y=\sum y^{2}$ $=\sum y^{3}=\sum y^{4}=0$, where $\sum y$ means $\sum_{i=1}^{9} P\left(x_{i}\right)$. If we assumie $P(x)$ to be a simple eighth degree polynomial, and attempt to set up the equations of condition directly, the work becomes involved. Hence we proceed as follows.

A theorem of Brioschi* states that, if $f(x)=0$ is of the $n$th degree, $n$ odd, there exist $(n-1) / 2$ polynomials in $x, \phi_{1}, \phi_{2}, \cdots, \phi_{(n-1) / 2}$, such that (1) each $\phi_{i}$ is of lower degree than $f(x)$, (2) the $\phi_{i}$ are linearly independent, (3) the $\phi_{i}$ possess the properties $\sum \phi_{i}=0 \dagger, \sum \phi_{i}{ }^{2}=0, \sum \phi_{i} \phi_{j}=0$, $(i, j=1,2, \cdots,(n-1) / 2, i \neq j),(4)$ the determination of the coefficients of the $\phi_{i}$ requires the solution of linear and quadratic equations only. Thus for $n=9$ there are 4 such functions, and if we set up the transformation

$$
y=c_{1} \phi_{1}+c_{2} \phi_{2}+c_{3} \phi_{3}+c_{4} \phi_{4}
$$

we have at once $\sum y=\sum y^{2}=0$, independent of the $c_{i}$. The two remaining equations of condition, $\sum y^{3}=0$ and $\sum y^{4}=0$, are clearly homogeneous equations of degree 3 and 4 respectively in the $c_{i}$, with coefficients which can be computed in terms of the coefficients of $f(x)=0$. If these two equations involved only two essential parameters their solution would, in general, require the solution of a 12 th degree equation, but the presence of an additional parameter allows it to be carried out without introducing any equation of degree higher than the fifth.

A well known theorem of Sylvester says that the general homogeneous cubic polynomial in four variables may, with

* Rendiconti del Istituto Lombardo, (2), vol. 20 (1887), pp. 364-370. Also his Opere, vol. 3, Milan, 1904, pp. 293-299.

$\dagger \phi_{i}$ means $\sum_{k-1}^{n} \phi_{i}\left(x_{k}\right)$, where the $x_{k}$ are the $n$ roots of $f(x)=0$. 
the aid of an equation of the fifth degree, be reduced to the canonical form $M_{1}{ }^{3}+M_{2}{ }^{3}+M_{3}{ }^{3}+M_{4}{ }^{3}+M_{5}{ }^{3}$, where the $M_{i}{ }^{3}$ are the roots of a quintic whose coefficients are rational in those of the given cubic polynomial, the $M_{i}$ are linear homogeneous functions of the four variables, and $M_{5}$ can be expressed as a linear function of the other $M_{i}$, say as $\sum_{i=1}^{4} v_{i} M_{i}$. The condition $\sum y^{3}=0$ can then be put in the form

(2) $M_{1}^{3}+M_{2}^{3}+M_{3}^{3}+M_{4}^{3}$

$$
+\left(v_{1} M_{1}+v_{2} M_{2}+v_{3} M_{3}+v_{4} M_{4}\right)^{3}=0,
$$

where each $M$ is a linear homogeneous function of $c_{1}, c_{2}, c_{3}$ and $c_{4}$. We wish to show that this equation can be satisfied in such a way as to leave one essential parameter undetermined.

In the exceptional case in which $v_{1}, v_{2}, v_{3}, v_{4}$ are all zero, (2) can be satisfied by setting $M_{2}=-M_{1}, M_{4}=-M_{3}$. Thus only two linear conditions are imposed on the $c_{i}$, and one essential parameter (that is, the ratio of two $c$ 's) remains unfixed.

In the general case, at least one $v$, say $v_{3}$, will be different from zero. We may also certainly rearrange subscripts so that the conditions $v_{1}=0, v_{2}{ }^{3}=v_{3}{ }^{3}$ do not hold simultaneously. Then make the following substitutions

$$
\left\{\begin{array}{l}
M_{1}=s+1, \\
M_{2}=a_{2} s+b_{2}, \\
M_{3}=\left(-v_{1}-v_{2} a_{2}\right) s / v_{3}+b_{3}, \\
M_{4}=b_{4} .
\end{array}\right.
$$

Equation (2) then becomes a non-homogeneous cubic in $s$, which can be satisfied identically in $s$ by proper choice of $a_{2}, b_{2}, b_{3}, b_{4}$. First, the coefficient of $s^{3}$ is

$$
1+a_{2}^{3}-\left(\frac{v_{1}+v_{2} a_{2}}{v_{3}}\right)^{3},
$$

and $a_{2}$ can be chosen so that this vanishes, under the restrictions on $v_{1}, v_{2}, v_{3}$, stated at the beginning of this paragraph. 
The coefficients of $s^{2}$ and $s$ are found to be respectively three times the quantities

$$
\begin{aligned}
& 1+a_{2}{ }^{2} b_{2}+a_{3}{ }^{2} b_{3}, \\
& 1+a_{2} b_{2}{ }^{2}+a_{3} b_{3}{ }^{2},
\end{aligned}
$$

where $a_{3}$ stands for $-\left(v_{1}+v_{2} a_{2}\right) / v_{3}$. Now $a_{2}$ can be chosen different from zero unless $v_{1}{ }^{3}=v_{3}{ }^{3}, v_{2}=0$. In this case, $a_{3}=-v_{1} / v_{3}$, and the coefficients of $s^{2}$ and $s$ can both be made to vanish by taking $b_{3}=-v_{1} / v_{3}$. Secondly, $a_{3}$ will be zero if $a_{2}=-v_{1} / v_{2}$; further, $1+a_{2}^{3}$ must equal zero, or $v_{1}^{3}=v_{2}^{3}$. In this case both the above coefficients will vanish if $b_{2}=-v_{1} / v_{2}$. Otherwise $a_{3}$ cannot equal zero unless $v_{1}=v_{2}=0$; then for $a_{2}=b_{2}=-1$ the coefficients of $s^{3}, s^{2}, s$ will all vanish. In the general case, both $a_{2}$ and $a_{3}$ will be different from zero, and $b_{2}$ and $b_{3}$ can obviously be determined so that the coefficients of $s^{2}$ and $s$ will vanish.

Finally, the term free of $s$ becomes

$$
1+b_{2}{ }^{8}+b_{3}{ }^{3}+b_{4}{ }^{3}+\left(v_{1}+v_{2} b_{2}+v_{3} b_{3}+v_{4} b_{4}\right)^{3} .
$$

To make this vanish requires simply the solution of a cubic in $b_{4}$, unless $1+v_{4}{ }^{3}=0$. If this condition holds, there remains a quadratic to solve unless $v_{1}+v_{2} b_{2}+v_{3} b_{3}=0$. However, if this last equation is satisfied, it may be combined with conditions already set up to show that $1+b_{2}{ }^{3}+b_{3}{ }^{3}$ must also equal zero. Thus the term free of $s$ still vanishes, and the coefficients of (3) have now been determined so that (2) vanishes identically in $s$.

Now since the $M_{i}$ are linear homogeneous functions of $c_{1}, \cdots, c_{4}$, the $c_{i}$ may be determined linearly in terms of $M_{1}, \cdots, M_{4}$. In general, this determination will be unique, but this will not be so if there is a linear relation between the $M_{i}$. By (3), the $c_{i}$ are given as linear, non-homogeneous functions of $s$. If now we substitute these values into the expression for $\sum y^{4}$, the final equation of condition, $\sum y^{4}=0$, becomes a non-homogeneous quartic equation in $s$, which can 
be solved for $s .^{*}$ This completes the proof of the theorem.

If $f(x)=0$ is of degree 7 or $8, \dagger$ there exist three $\phi$-functions having the properties of Brioschi's theorem. We then set up a transformation similar to (1), and the equations $\sum y^{3}=0, \sum y^{4}=0$ are homogeneous equations in three parameters. The determination of $c_{1}, c_{2}, c_{3}$ will, in general, lead to an equation of the 12 th degree, as was pointed out above.

If $f(x)=0$ is of degree 6 it does not seem possible to lower materially the maximum 24 which was obtained in the second paragraph.

The University of California at Los Angeles

\title{
SOME PROPERTIES OF MULTI-COHERENT CONTINUA
}

\author{
BY W. A. WILSON
}

1. In a recent paper§ C. Kuratowski gives the following definitions:

A continuum $C$ is called uni-coherent (or $n$-coherent) if for every decomposition of $C$ into two continua $K$ and $L$, where $C \neq K \neq L, K \cdot L$ has one (or $n$ ) components. A continuum $C$ is called multi-coherent, if $K \cdot L$ is not connected.

The properties of such continua are later developed in some detail. Among the theorems proved are the following.

* The very exceptional case in which the coefficients of $s^{4}, s^{3}, s^{2}, s$ all vanish, while the term free of $s$ does not vanish, may be handled by introducing homogeneous parameters in (3), that is, by putting $M=s+t$, $M_{2}=a_{2} s+b_{2} t$, etc. For the present case, $t$ must be zero.

$\dagger$ Although Brioschi's theorem does not mention equations of even degree, $(n-1) / 2 \phi$-functions can be set up for an equation of even degree $n+1$ just as they can for an equation of odd degree $n$, and in different ways as well.

$\ddagger$ Presented to the Society, October 27, 1928.

$\S$ C. Kuratowski, Sur la structure des frontières communes d deux regions, Fundamenta Mathematicae, vol. 12, pp. 20-42. 